\title{
Resonant intersubband polariton-LO phonon scattering in an optically pumped polaritonic device
}

\author{
J-M. Manceau ${ }^{1,{ }^{*}}$, N-L. $\operatorname{Tran}^{1}$, G. Biasiol ${ }^{2}$, T. Laurent ${ }^{1}$, I Sagnes $^{1}$, G. Beaudoin ${ }^{1}$, \\ S. De Liberato ${ }^{3}$, I. Carusotto ${ }^{4}$, R. Colombelli ${ }^{1, \#}$ \\ ${ }^{1}$ Centre de Nanosciences et de Nanotechnologies, CNRS UMR 9001, Université Paris-Sud, Université \\ Paris-Saclay, C2N - Orsay, 91405 Orsay cedex, France \\ ${ }^{2}$ Laboratorio TASC, CNR-IOM, Area Science Park, S.S. 14 km 163.5 Basovizza, I-34149 Trieste, Italy, \\ ${ }^{3}$ Department of Physics and Astronomy, University of Southampton, Highfield, Southampton, UK \\ ${ }^{4}$ INO-CNR BEC Center and Dipartimento di Fisica, Universita di Trento, I-38123 Povo, Italy
}

We report experimental evidence of longitudinal optical (LO) phonon-intersubband polariton scattering processes under resonant injection of light. The scattering process is resonant with both the initial (upper polariton) and final (lower polariton) states and is induced by the interaction of confined electrons with longitudinal optical phonons. The system is optically pumped with a mid-IR laser tuned between $1094 \mathrm{~cm}^{-1}$ and $1134 \mathrm{~cm}^{-1}(\lambda=9.14 \mu \mathrm{m}$ and $\lambda=8.82$ $\mu \mathrm{m})$. The demonstration is provided for both GaAs/AlGaAs and InGaAs/AlInAs doped quantum well systems whose intersubband plasmon lies at $\approx 10 \mu \mathrm{m}$ wavelength. In addition to elucidating the microscopic mechanism of the polariton-phonon scattering, that is found to differ substantially from the standard single particle electron-LO phonon scattering mechanism, this work constitutes the first step towards the hopefully forthcoming demonstration of an intersubband polariton laser. 
Since their first theoretical prediction two decades ago [1], intersubband (ISB) polaritons have been subject to abundant theoretical and experimental work. Shortly after their first experimental demonstration [2], it was understood that they offer the particularity to access a new regime of interaction, namely the ultra-strong coupling regime, which could initiate interesting quantum phenomena when modulated in time [3,4]. These theoretical predictions have been driving the experimental field for years towards the demonstration of unprecedented Rabi-splitting values [5-9]. The other key aspect of intersubband polaritons is their bosonic nature which, as for their excitonic counterpart [10], enables a regime of final state stimulation either via longitudinal optical phonons $\left(\mathrm{LO}_{\mathrm{ph}}\right)$ scattering or via polaritonpolariton scattering [11,12]. As a crucial new feature of ISB polariton devices, reference [13], that details a roadmap towards an ISB polariton laser, also points out that the upper density limit for bosonic behavior of ISB polaritons is not rigidly fixed - as is the case of the Mott density for excitons - but it can be engineered by design to a large extent with the electronic doping. This observation suggests that ISB polariton lasers should be relatively high output power devices.

To date, most - if not all - of the attempts to implement intersubband polaritonic light emitting devices were led under electrical pumping [14-19]. Such approach is hampered by the presence of the dark states of the ISB plasmon, that do not couple to the electro-magnetic field. The number of dark states being important, only a small fraction of the injected electrons tunnel into the bright polaritonic states and the efficiency of the process is dramatically reduced [20]. Optical pumping of intersubband polaritonic systems has instead concentrated on time domain studies demonstrating the ultrafast switching and bleaching of the strong coupling regime $[21,22]$. These experiments employ femtosecond-pulses tuned at the interband transition of the semiconductor quantum wells. These pulses induce carriers in the system - via electron-hole generation - but do not couple to the ISB polaritons. To date, and to the best of our knowledge, optical pumping directly at the intersubband polariton energies has never been reported. Yet this is the most effective manner to selectively inject energy into the bright polaritonic states.

In this letter we experimentally investigate the coupling between ISB polaritons and LO phonons. In particular, we report evidence of a scattering process that is resonant with both the initial (upper polariton, UP) and final (lower polariton, LP) states. The system is pumped with a narrowband, resonant optical excitation at the UP energy. The $\mathrm{LO}_{\mathrm{ph}}$ origin of the scattering mechanism is attested by the frequency of the scattered light that is red-shifted from 
the pump by $293.5 \mathrm{~cm}^{-1}$ in a GaAs/AlGaAs quantum well (QW) system, and by $271 \mathrm{~cm}^{-1}$ in an InGaAs/AlInAs QW system. Within the resolution limit of the measurement, the emitted/scattered light is spectrally as narrow as the pump laser, and - in agreement with its resonant character - its amplitude is proportional to the absorption of the injection state. The intensity of the scattered light is in fair agreement with theoretical calculations of the ISB polariton-LO phonon coupling along the lines of [11], which leads to an optimistic quantitative estimate for the laser threshold.

We have developed in recent years microcavity resonators that offer an energy minimum at $k=0$ with a positive, parabolic dispersion for transverse magnetic (TM) polarized modes $[23,24]$. When coupled to an ISB plasmon, it permits to translate to the mid-IR spectral region the peculiar dispersion of excitonic polaritons, that has been the key enabling tool behind the demonstration of stimulated scattering and lasing in these systems. We have shown that this resonator is operational over a very large frequency range and it is also compatible with electrical injection $[19,25]$. Most importantly, it suits optical pumping experiments as light can be injected at a different angle from the collection angle $(\theta=0)$ hence minimizing stray light coming from the pump onto the detector. Figure 1a depicts the device under study. The top grating (period $\Lambda=4.26 \mu \mathrm{m}$ with filling factor of $72 \%$ ) couples the impinging light by the surface and confines it within the active core volume. The metal-metal configuration allows maximum overlap of the electromagnetic field with the active core (sample HM3872) that consists in a 36-period repetition of 8.3-nm-thick GaAs QWs separated by 20-nm-thick $\mathrm{Al}_{0.3} \mathrm{Ga}_{0.7} \mathrm{As}$ barriers. The nominal surface doping per $\mathrm{QW}\left(\mathrm{n}_{2 \mathrm{D}}=4.4 \times 10^{12} \mathrm{~cm}^{-2}\right)$ is introduced as $\delta$-layers in the center of the barriers. The absorptions of the ISB transition at $300 \mathrm{~K}$ and $78 \mathrm{~K}$ are available elsewhere [26].

The presence of the two polaritonic eigenstates, characteristic of the strong coupling regime, is probed in the frequency domain using a Fourier Transform Infrared (FTIR) spectrometer. From the spectra recorded at different angles (from $13^{\circ}$ to $61^{\circ}$, in $2^{\circ}$ steps, using a commercial, motorized angle reflection unit) the polaritonic dispersion can be readily obtained as explained in [23, 24]. It is crucial to assess the spectral position of the LP branch at normal incidence, as it is where the polaritons, optically injected in the upper branch, shall be scattered. We probed them using a different grating and electric field orientation: the reflectivity minima recorded at $300 \mathrm{~K}$ are marked as red stars on the dispersion curve in Fig. 1b. The same measurement performed at $78 \mathrm{~K}$ is reported in Fig. $2 \mathrm{a}$ (red curves): it reveals as 
expected increase of the polariton branches separation, mainly governed by the blue shift of the UP by $30 \mathrm{~cm}^{-1}$.

All the gathered information on the polaritonic dispersion of sample HM3872 permits to precisely identify the correct incidence angle to induce a polariton-LO phonon scattering process between the upper polariton injection and the lower polariton final states. From the literature [27], the $\mathrm{LO}_{\mathrm{ph}}$ energy at $78 \mathrm{~K}$ and at $k=0$ is $294 \mathrm{~cm}^{-1}$ : this places the resonant pumping configuration at an incidence angle of 41 degrees, with a LP at $824 \mathrm{~cm}^{-1}$ and an UP at $1118 \mathrm{~cm}^{-1}$.

Figure 1a shows the experimental approach. The incident laser light is coupled in through an anti-reflection (AR) coated $\mathrm{ZnSe}$ lens ( $\mathrm{f} \mathrm{\# 2}$ ) and the specular component of the reflection is measured using a power-meter. The scattered light is collected at normal incidence with an AR coated ZnSe lens (f \#1.5) and analyzed with an FTIR spectrometer equipped with a liquid-nitrogen cooled Mercure Cadmium Telluride (MCT) detector. The pump laser source is a commercial quantum cascade laser (QCL) from Daylight solutions (MirCat), tunable from 1026 to $1140 \mathrm{~cm}^{-1}$ with a maximum average output power in pulsed mode (100 kHz, $\left.2 \mu \mathrm{s}\right)$ of $111 \mathrm{~mW}$ (peak power within the pulse is 5 times more). Using a knife-edge technique, we estimated the beam waist at the focal point to be $170 \mu \mathrm{m}\left(1 / \mathrm{e}^{2}\right)$. The average estimated pump intensity can therefore reach up to $500 \mathrm{~W} / \mathrm{cm}^{2}\left(2.5 \mathrm{~kW} / \mathrm{cm}^{2}\right.$ peak power).

It is possible to verify that the pump angle on the sample is correct. We record the $0^{\text {th }}$ order reflected light from the sample while tuning the laser wavenumber in $2 \mathrm{~cm}^{-1}$ steps. The reflectivity is obtained by dividing the data with the one recorded on a plane gold mirror. Figure 2a (blue curve) shows the reflectivity of the sample, across the pump laser tuning range, for an incidence angle of $41^{\circ}$ and at $78 \mathrm{~K}$ : we can clearly identify the UP state. We therefore fix the pump wavelength at the maximum absorption of the injection (UP) state $\left(1118 \mathrm{~cm}^{-1}\right)$ and record, using a synchronous detection scheme, the scattered light collected at normal incidence for $77 \mathrm{~mW}$ of average power impinging on the sample. Given the $75 \%$ absorption of the sample at this angle and frequency, we estimate that $58 \mathrm{~mW}$ of average power are injected in the upper polariton state. To reduce the amount of stray light coming from the pump beam diffused at the surface of the sample, we have placed a long wave pass filter in front of the detector ( $30 \mathrm{~dB}$ attenuation at the pump wavelength).

Figure $2 \mathrm{~b}$ shows the experimental spectrum obtained in step-scan. One can clearly observe the presence of two peaks, one corresponding to the laser pump diffused by the imperfections 
of the grating and a second lower peak separated by the energy of one $\mathrm{LO}_{\mathrm{ph}}\left(293 \mathrm{~cm}^{-1}\right)$ from the pump signal. The two peaks match with the maximum absorption of both injection and lower states. We have increased the resolution of the measurement down to $0.5 \mathrm{~cm}^{-1}$ allowing a more precise measurement of the $\mathrm{LO}_{\mathrm{ph}}$ separation at $293.5 \mathrm{~cm}^{-1}$. Interestingly, the scattered light is a replica of the injected laser light and no convolution with the $\mathrm{LO}_{\mathrm{ph}}$ lineshape is observable. Within this resolution limited scan, we can extract a full width at half maximum (FWHM) for the scattered light of $1 \mathrm{~cm}^{-1}$.

We then study the dependence of the scattered light on the injection laser frequency. The emission spectra for different pump wavenumbers were recorded in steps of $8 \mathrm{~cm}^{-1}$. The integration time of the measurements were kept similar. The data are reported in Fig. 4. They reveal that the amount of scattered/emitted light is directly proportional to the absorption at the injection frequency, and that the $\mathrm{LO}_{\mathrm{ph}}$ energy separation is strictly respected as we change the pump wavenumber. Since we are collecting the scattered light within a large angular cone $\left( \pm 19^{\circ}\right)$, the influence on the final state is weak, especially since the latter is relatively flat over this cone of angles and broad in frequency.

As a last piece of evidence of the $\mathrm{LO}_{\mathrm{ph}}$-related scattering mechanism, we have led the same experiments with a polaritonic sample based on the InGaAs/AlInAs system lattice matched on InP. The active core (sample InP1614) consists in 35-periods repetition of $10.5 \mathrm{~nm}$ thick InGaAs QWs separated by 15-nm-thick AlInAs barriers. The nominal doping of the sample $\mathrm{n}_{3 \mathrm{~d}}=1 \times 10^{18} \mathrm{~cm}^{-3}$ is introduced as bulk within the wells. From the literature, the $\mathrm{LO}_{\mathrm{ph}}$ in this system is expected to be around in the $270-274 \mathrm{~cm}^{-1}$ range, depending on the exact alloy concentration [28]. Our measurements show a separation between the injected and scattered light of $271 \mathrm{~cm}^{-1}$ at $78 \mathrm{~K}$ as presented on Figure S1 of the Supplementary Material. It presents a typical spectrum along with the measurement of the GaAs/AlGaAs sample. In order to highlight the difference in phonon separation for the two systems, we have plotted the frequency axis subtracting the pump frequency. One can clearly observe a difference of 22.5 $\mathrm{cm}^{-1}$ between the spectral positions of the scattered light in the two systems. The full dispersion of the sample InP1614, as well the reflectivity of the final and injection states measured at $78 \mathrm{~K}$, are also available in Supplementary Material.

These results have been obtained with an incident peak power of $\approx 1.25 \mathrm{~kW} / \mathrm{cm}^{2}$. This is an extremely low power with respect to the only similar literature available, that is the works on the quantum fountain laser (QFL) and Intersubband Raman Laser (IRL) reported at the end of 
the nineties $[29,30]$. These lasers, operating in the weak light-matter coupling regime, rely on population inversion within a 3 levels system present in asymmetrically coupled quantum wells. In those works, no emission signal was ever detected for incident powers below approximately $1 \mathrm{MW} / \mathrm{cm}^{2}$. Here, we are able to observe emission for incidence powers that are lower by at least three orders of magnitude, which is a remarkable result. However, the important question is: how far are we from the onset of a stimulated process?

A quantitative estimate for the intensity of the scattered light can be obtained by deriving from the usual electron-phonon Frölich Hamiltonian the matrix element for the LO phononISB plasmon coupling. A simple Fermi golden rule calculation generalizing the calculations in Ref. [31], predicts the following spontaneous, angle-integrated phonon scattering rate:

$$
\Gamma_{p h}=\frac{\pi \omega_{L O} e^{2} k_{f} L_{Q W} f\left|u_{I S B}^{i} u_{I S B}^{f}\right|^{2}}{\hbar v_{f}^{g r} \epsilon_{\rho}}
$$

where $\omega_{L O}$ is the LO phonon angular frequency, $k_{i, f}$ are the wave-vectors of the initial and final ISB polariton states, $v_{f}^{g r}$ is their group velocity (assuming an isotropic polariton dispersion around a minimum at $k=0$ ), $\mathrm{L}_{\mathrm{QW}}$ is the $\mathrm{QW}$ width, $u^{i, f}{ }_{I S B}$ are the ISB plasmon Hopfield weights in the initial and final ISB polaritons, $\varepsilon_{\rho}=\left[\varepsilon_{\infty}^{-1}-\varepsilon_{s}^{-1}\right]^{-1}$ is the relative dielectric constant due to phonons, and $f$ is a numerical factor of order 0.03 summarizing the intrasubband nature of the scattering process [31]. Inserting the actual figures for our device, we find a $\sim 10^{-7}$ branching ratio for phonon-polariton scattering compared to radiative and non-radiative losses. This value is in reasonable agreement with our experimental observations of the efficiency of the process. With an average power of $58 \mathrm{~mW}$ injected within the upper polaritonic state - based on the detector responsivity and the various losses induced by the measurement chain - we reach an estimated efficiency of $6 \times 10^{-9}$ for the measured scattered signal.

Formula (1) yields an ISB polariton/LO-phonon scattering time in the $\mu$ s range, a value orders of magnitude longer than the ps-range scattering time of electron-phonon scattering typically found in QCL devices, i.e. the scattering of an electron from the second to the first subband in a semiconductor QW. This dramatic difference has a twofold origin. On one hand, it is partly a consequence of the much lower polariton mass compared to the electronic one. On the other hand, and most importantly, the single particle electron-phonon process in a QW is clearly of intersubband nature, while the the polariton-phonon scattering is essentially an 
intrasubband process, which reduces the matrix element of the process. To our knowledge, it is the first time that this fundamental peculiarity of ISB polariton/LO phonon scattering is highlighted.

To go beyond the spontaneous scattering rate and estimate the threshold for the onset of bosonic stimulation, we take advantage of our observation of a very long lifetime of the phonon mode and view the process as an upper ISB polariton being coherently downconverted into a signal-idler pair formed by a lower ISB polariton plus a phonon. Using the usual theory of parametric oscillation in planar devices [32], we can extract a value for the threshold:

$$
I_{t h}=\frac{\hbar^{2} \Gamma_{L O} \Gamma_{U P} \Gamma_{L P} \omega_{U P} \varepsilon_{\rho}}{4 \pi\left|u_{I S B}^{i}\right|^{2}\left|u_{I S B}^{f}\right|^{2} \omega_{L O} f e^{2} L_{Q W}}
$$

Plugging into this equation actual values of our structure $\left(\hbar \Gamma_{u p} \sim 4 \mathrm{meV}, \hbar \Gamma_{l p} \sim 7 \mathrm{meV}\right.$ and $\hbar \Gamma_{L O}<0.36 \mathrm{meV}$ (LO-phonon Q-factor around 100) and $75 \%$ of absorbed light within the upper polariton state), we find a threshold intensity of the order of $70 \mathrm{~kW} / \mathrm{cm}^{2}$, in agreement with the conclusions of Ref. [11] and, most remarkably, just a couple of orders of magnitude higher than the one reported here. With a reasonable focus as presented above, such level of intensities correspond to incident average powers of $\approx 20 \mathrm{~W}$, that could be reached with pulsed $\mathrm{CO}_{2}$ lasers or using energetic pulses emitted from an OPO system. Alternatively, pump-probe techniques could be used to populate the final state and facilitate the stimulated parametric scattering regime as elegantly demonstrated for exciton-polaritons [33]. An important difference compared to the excitonic case is however that the idler mode consists here of a phonon rather than another polariton, which makes the development of a coherently emitting device even more intriguing.

In conclusion, we have demonstrated spontaneous resonant ISB polariton - LO phonon scattering in an optically pumped device operating in the strong coupling regime between light and matter. Both the spectral position and the intensity of the scattering process are consistent with the theoretical predictions for GaAs and InGaAs based systems. Although the intrasubband nature of the electron-phonon process underlying the observed polariton-phonon scattering dramatically quenches its rate as compared to standard electron-phonon processes in QCLs, we predict an optimistic value in the $70 \mathrm{~kW} / \mathrm{cm}^{2}$ for the polariton lasing threshold intensity. We finally anticipate that the laser device might have practical application as a coherent source not only of Mid-IR light, but also of LO phonons. 


\section{$\underline{\text { Supplementary material: }}$}

See supplementary material at ... for the recorded emission spectra within the InGaAs/AlInAs (InP1614). Additional information on the recorded dispersion of the sample at $300 \mathrm{~K}$ as well as the reflectivity curve of the final and injection state at $78 \mathrm{k}$ are also presented there.

We thank S. Pirotta, A. Bousseksou and F. Julien for useful discussions. We acknowledge financial support from the European Union FET-Open grant MIR-BOSE 737017, and from the European Research Council (IDEASERC) ("GEM") (306661). This work was partly supported by the French RENATECH network. 


\section{References}

*E-mail: jean-michel.manceau@u-psud.fr

\#E-mail: raffaele.colombelli@u-psud.fr

[1] A. Liu, Physical Review B 55, 7101 (1997).

[2] D. Dini, R. Köhler, A. Tredicucci, G. Biasiol, and L. Sorba, Physical Review Letters 90, 116401 (2003).

[3] C. Ciuti, G. Bastard, and I. Carusotto, Physical Review B 72, 115303 (2005).

[4] C. Ciuti and I. Carusotto, Physical Review A 74, 033811 (2006).

[5] E. Dupont, J. A. Gupta, and H. C. Liu, Physical Review B 75, 205325 (2007).

[6] A. A. Anappara, S. De Liberato, A. Tredicucci, C. Ciuti, G. Biasiol, L. Sorba, and F. Beltram, Physical Review B 79, 201303 (2009).

[7] Y. Todorov, A. M. Andrews, R. Colombelli, S. De Liberato, C. Ciuti, P. Klang, G. Strasser, and C. Sirtori, Physical Review Letters 105, 196402 (2010).

[8] M. Geiser, F. Castellano, G. Scalari, M. Beck, L. Nevou, and J. Faist, Physical Review Letters 108, 106402 (2012).

[9] B. Askenazi, A. Vasanelli, A. Delteil, Y. Todorov, L. C. Andreani, G. Beaudoin, I. Sagnes, and C. Sirtori, New Journal of Physics 16, 043029 (2014).

[10] D. Bajoni, Journal of Physics D: Applied Physics 45, 313001 (2012).

[11] S. De Liberato and C. Ciuti, Physical Review Letters 102, 136403 (2009).

[12] S. De Liberato, C. Ciuti, and C. C. Phillips, Physical Review B 87, 241304(R) (2013).

[13] R. Colombelli and J.-M. Manceau, Physical Review X 5, 011031 (2015).

[14] R. Colombelli, C. Ciuti, Y. Chassagneux, and C. Sirtori, Semiconductor Science and Technology 20, 985 (2005).

[15] L. Sapienza, A. Vasanelli, R. Colombelli, C. Ciuti, Y. Chassagneux, C. Manquest, U. Gennser, and C. Sirtori, Physical Review Letters 100, 136806 (2008).

[16] P. Jouy, A. Vasanelli, Y. Todorov, L. Sapienza, R. Colombelli, U. Gennser, and C. Sirtori, Physical Review B 82, 045322 (2010).

[17] A. Delteil, A. Vasanelli, P. Jouy, D. Barate, J. C. Moreno, R. Teissier, A. N. Baranov, and C. Sirtori, Physical Review B 83, 081404 (2011).

[18] M. Geiser, G. Scalari, F. Castellano, M. Beck, and J. Faist, Applied Physics Letters 101, 141118 (2012).

[19] D. Chastanet, J.-M. Manceau, T. Laurent, A. Bousseksou, G. Beaudoin, I. Sagnes, and R. Colombelli, Applied Physics Letters 110, 081108 (2017).

[20] S. De Liberato and C. Ciuti, Physical Review B 79, 075317 (2009).

[21] G. Gunter et al., Nature 458, 178 (2009).

[22] S. Zanotto, R. Degl'Innocenti, J.-H. Xu, L. Sorba, A. Tredicucci, and G. Biasiol, Physical Review B 86, 201302 (2012).

[23] J.-M. Manceau, S. Zanotto, T. Ongarello, L. Sorba, A. Tredicucci, G. Biasiol, and R. Colombelli, Applied Physics Letters 105, 081105 (2014).

[24] J. M. Manceau, S. Zanotto, I. Sagnes, G. Beaudoin, and R. Colombelli, Applied Physics Letters 103, 091110 (2013).

[25] T. Laurent, J.-M. Manceau, E. Monroy, C. B. Lim, S. Rennesson, F. Semond, F. H. Julien, and R. Colombelli, Applied Physics Letters 110, 131102 (2017). 
[26] J. M. Manceau, G. Biasiol, N. L. Tran, I. Carusotto and R. Colombelli, Physical Review B 96 (23), 235301 (2017).

[27] S. Adachi, Journal of Applied Physics 58 (3), R1-R29 (1985).

[28] T. P. Pearsall, R. Carles, and J. C. Portal, Applied Physics Letters 42, 436 (1983).

[29] O. Gauthier-Lafaye, P. Boucaud, F. H. Julien, S. Sauvage, S. Cabaret, J. M. Lourtioz, V. Thierry-Mieg, and R. Planel, Applied Physics Letters 71, 3619 (1997).

[30] H. C. Liu, I. W. Cheung, A. J. SpringThorpe, C. Dharma-wardana, Z. R. Wasilewski, D. J. Lockwood, and G. C. Aers, Applied Physics Letters 78, 3580 (2001).

[31] R. Ferreira and G. Bastard, Physical Review B 40 (2), 1074-1086 (1989).

[32] M. Wouters and I. Carusotto, Physical Review B 75 (7), 075332 (2007).

[33] P. G. Savvidis, J. J. Baumberg, R. M. Stevenson, M. S. Skolnick, D. M. Whittaker, and J. S. Roberts, Physical Review Letters 84, 1547 (2000). 
Figure 1: (a) Schematic representation of the experimental geometry. (b) Experimentally recorded reflectivity minima of the polaritonic sample (HM3872) at 300K. The two polaritonic branches are clearly separated and the reflectivity minima at $0^{\circ}$ degree are marked with stars as they required a different experimental configuration to be measured.

Figure 2: (a) Spectral reflectivity of the sample at normal incidence (red lines) and at $41^{\circ}$ incidence (blue line). The LP state evident in the red line corresponds to the final state, while the UP state in the blue line corresponds to the injection state. Both measurements were performed at $78 \mathrm{~K}$. The injection state (blue line) is recorded step by step using the QCL and its specular reflection. The final state (red line) is recorded within the FTIR. (b) Emission spectrum of sample HM3872 under resonant light injection. The laser incident power is $58 \mathrm{~mW}$. The measurement was performed in step-scan configuration at a resolution of $4 \mathrm{~cm}^{-1}$ and a temperature of $78 \mathrm{~K}$. One can clearly observe the correspondence with the injection and final state, separated by one $\mathrm{LO}_{\mathrm{ph}}$.

Figure 3: Emission spectra of sample HM3872 under resonant optical pumping recorded with increasing spectral resolutions (from the top: $4 \mathrm{~cm}^{-1}, 1 \mathrm{~cm}^{-1}, 0.5 \mathrm{~cm}^{-1}$ ). Within the resolution limit of the spectrometer, it allows to accurately measure the LO phonon energy at $293.5 \mathrm{~cm}^{-1}$, very close to the bulk value in GaAs.

Figure 4: Emission spectra of sample HM3872 for different values of the pump laser wavenumber. The scattered light strictly follows the injected one and it is always separated by one $\mathrm{LO}_{\mathrm{ph}}$. Note that the amount of scattered/emitted light is directly proportional to the absorption at the injection frequency. 
(a)

From QCL To FTIR
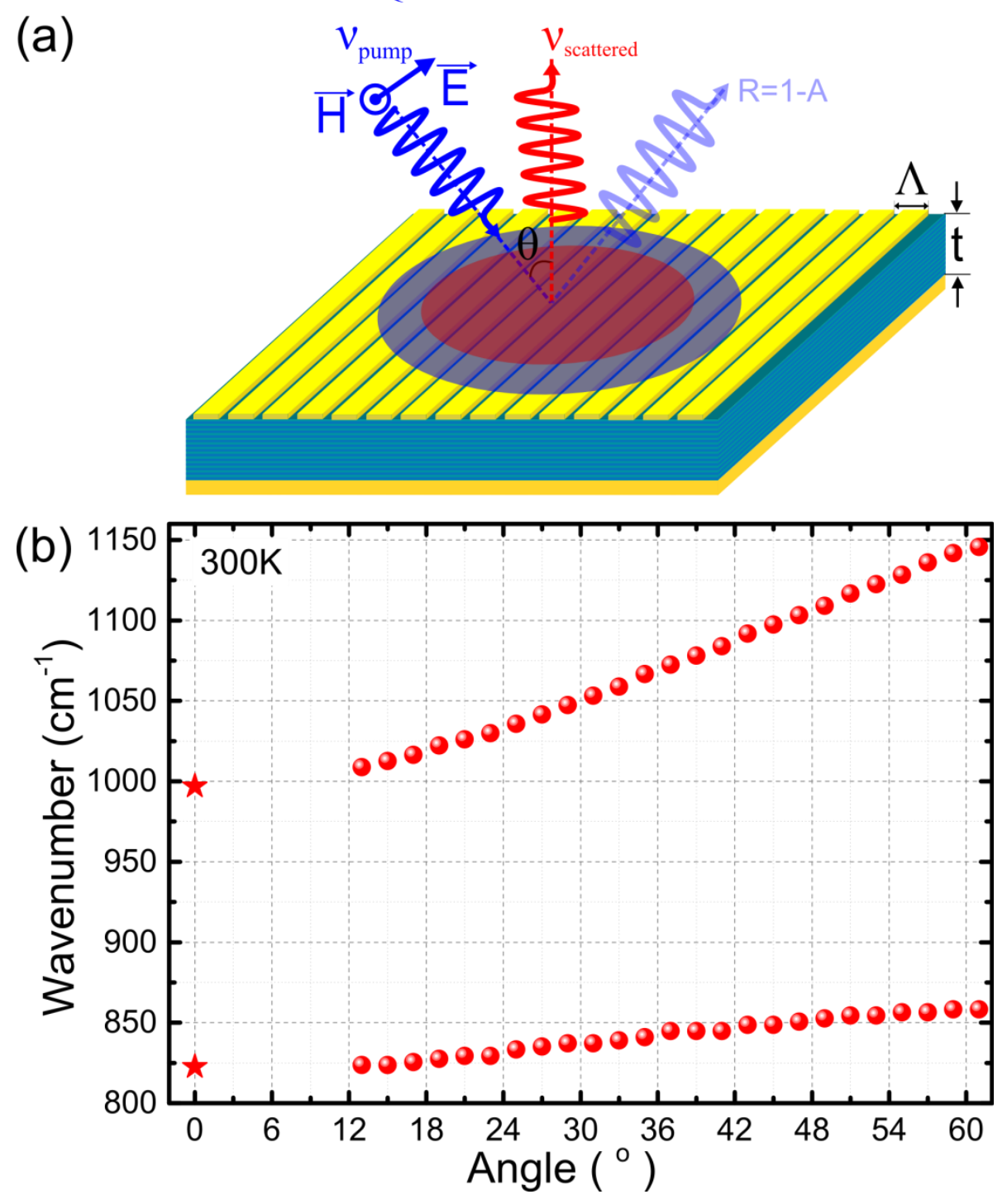

Figure 1: JM Manceau et al. 


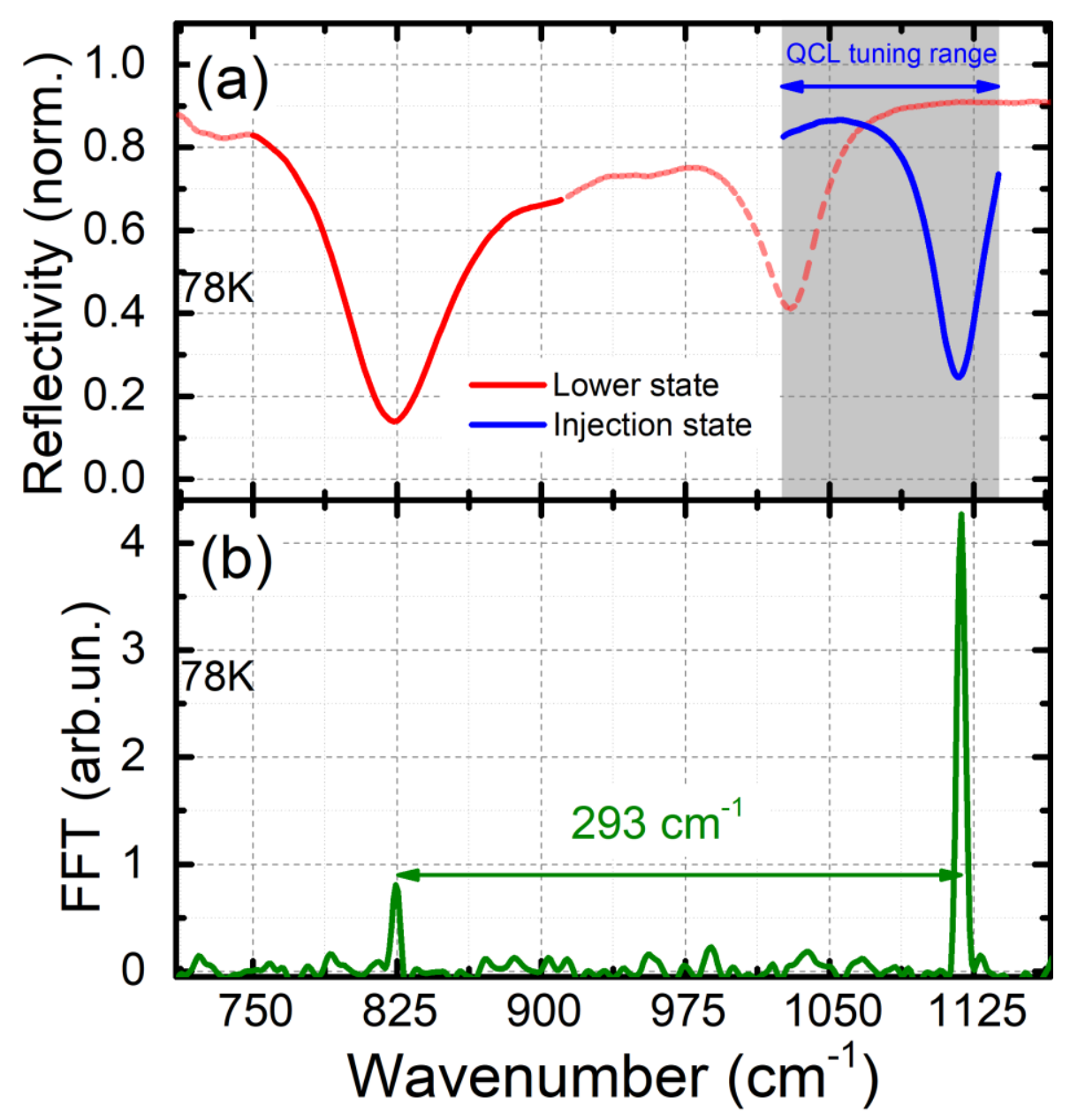

Figure 2: JM Manceau et al. 


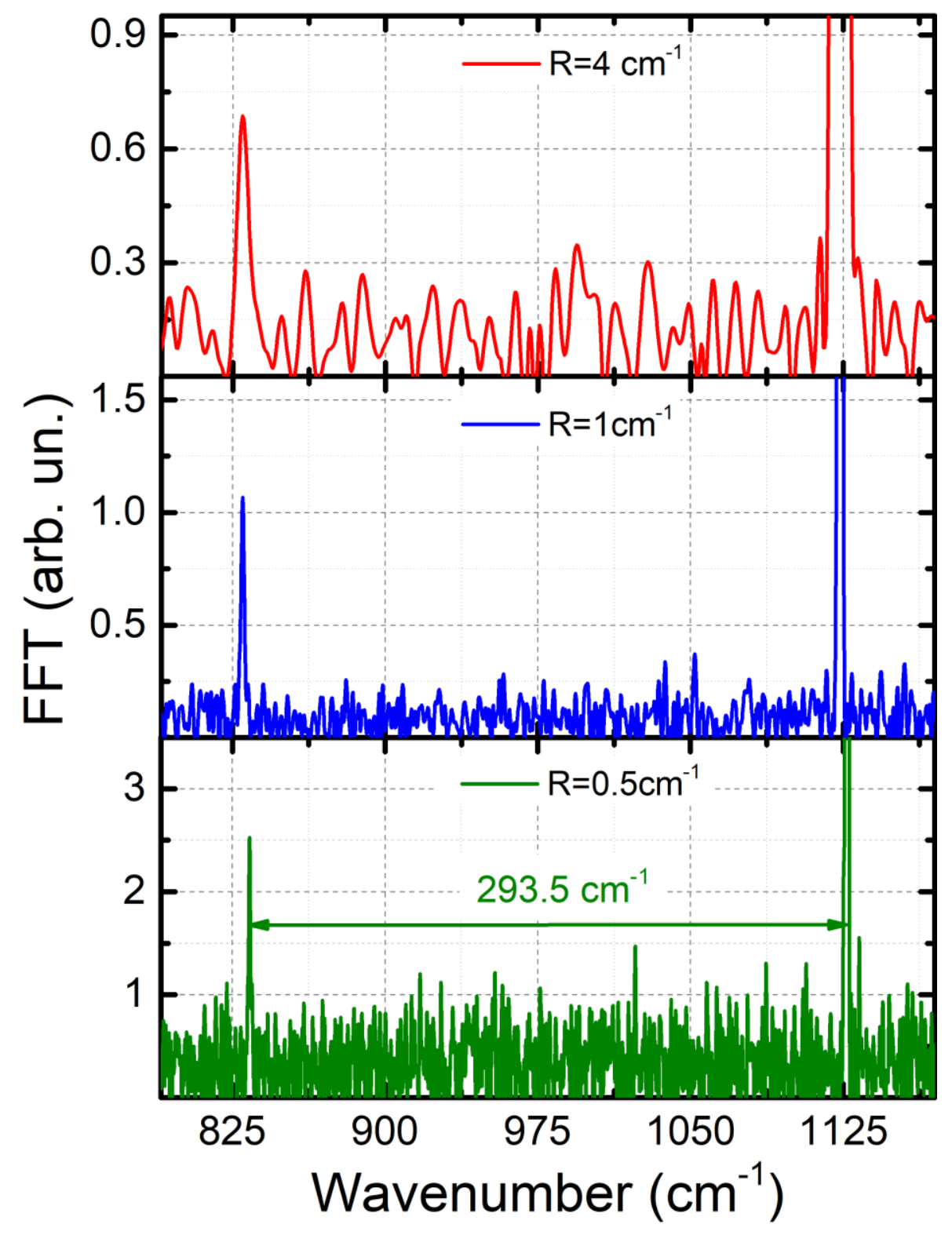

Figure 3: JM Manceau et al. 


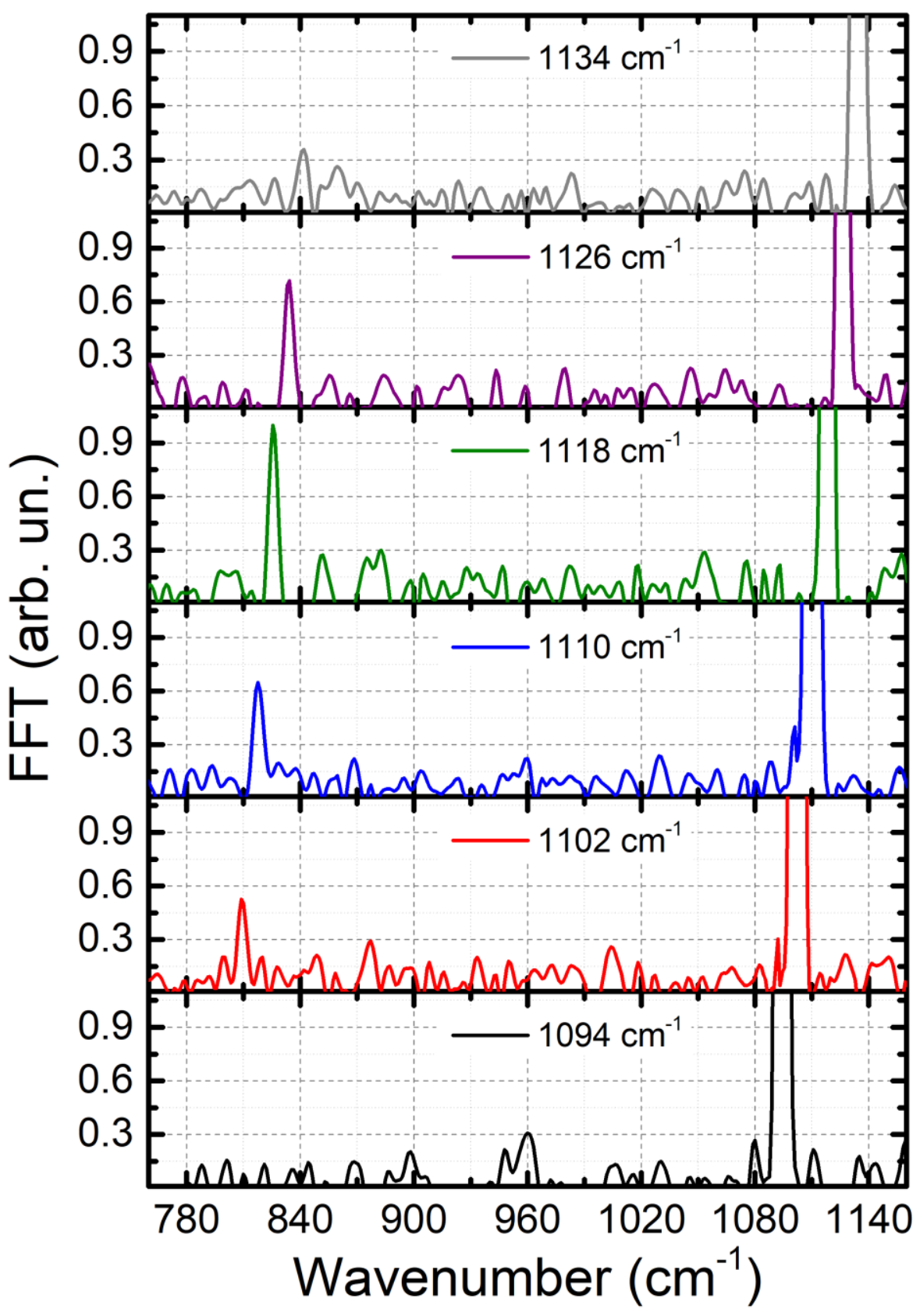

Figure 4: JM Manceau et al. 